\title{
Soap and water prophylaxis for limiting genital ulcer disease and HIV-1 infection in men in sub-Saharan Africa
}

\author{
Nigel O’Farrell
}

\begin{abstract}
In general, East, Central and Southern Africa appear to be worse affected by HIV-1 infection than West Africa. So far there is little evidence to suggest that differences in either sexual behaviour or numbers of sexual partners could account for this disparity. Two risk factors in men for acquiring HIV-1, that tend to vary along this geographical divide, are lack of circumcision and genital ulcer disease (GUD) which are much less common in West Africa. Although uncircumcised men with GUD are an important high frequency HIV-1 transmitter core group, few interventions have targeted such individuals. Given the recent expansion in AIDS-related technologies, is it possible that methods effective in limiting GUD in the preantibiotic era have been overlooked? During the first and second world wars, chancroid, the commonest cause of GUD in Africa today, was controlled successfully with various prophylactics including soap and water. Many parts of Africa are undergoing social upheaval against a background of violence, and in this environment soap and water prophylaxis would now seem to merit re-evaluation as an intervention for preventing both GUD and HIV-1 in uncircumcised men. By facilitating healing of traumatic, inflammatory and infected penile lesions, pre- and post-exposure prophylaxis with soap and water could be a cheap and effective method for decreasing the risks of acquiring GUD and HIV in this vulnerable group of uncircumcised men.
\end{abstract}

(Genitourin Med 1993;69:297-300)

Current global estimates of the numbers of persons infected with HIV-1 indicate a much larger problem in sub-Saharan Africa than elsewhere. Factors associated with the rapid spread of HIV-1 infection in Africa include poverty, lack of education, a high prevalence of sexually transmitted diseases (STD) and prostitution. ${ }^{1}$ In Africa the heterosexual transmission of HIV-1 is highly efficient and many women become infected despite limited numbers of sexual partners. ${ }^{2}$ Interventions that reduce this rate could have a significant impact in limiting the spread of HIV-1 and are most cost effective if introduced as early as possible in the time course of the epidemic. $^{3}$ Initial prevention programmes were directed at whole populations but subsequently high-frequency STD transmitter core groups have been targeted for HIV related education and information. Interventions concentrated upon both prostitutes and their clients $^{45}$ and truck drivers ${ }^{6}$ have proved to be cost effective in limiting the spread of HIV infection. ${ }^{4}$ However, as the epidemic progresses and spreads into the general population, other core groups, perhaps not so readily identifiable will need to be sought. Because HIV-1 is transmitted more easily from male to female than vice versa and, outside of the established core groups, men in Africa probably have more sexual partners than women, ${ }^{7}$ it would seem logical to target those men whose sexual practices and behaviour put them at risk of acquiring $\mathrm{HIV}-1$ infection.

Within Africa the spread of HIV-1 has not been uniform. Marked variation in the prevalence of HIV-1 occurs both between countries with common borders and in different parts of the same country. For example, the current numbers of reported cases ${ }^{8}$ of AIDS $/ 100,000$ in Uganda is 44.9 and in neighbouring Sudan 0.5. Substantial differences are also found both between Malawi 48.3 and Mozambique 0.6 and Ivory Coast 26.6 and Liberia $0 \cdot 0$. In South Africa the prevalence of HIV-1 amongst antenatal clinic attenders on the East Coast in Natal/ KwaZulu at $2 \cdot 87 \%$ is nearly 8 times greater than on the West Coast in the Cape $(0.37 \%) .{ }^{9}$ Overall, countries in West Africa, with the exception of the Ivory Coast and possibly Ghana, generally have much lower rates of HIV-1 infection than Eastern, Central and Southern Africa. ${ }^{8}$ What factors may account for this variation?

Differences between the times of introduction of new cases into a country are relevant but would not explain the current rapid increase in heterosexual cases of HIV-1 in South Africa where the infection only entered the heterosexual population relatively recently in 1987-8.10 Assessment of rates of sexual partner change or variation in the numbers of unprotected sex acts are important in determining the relative risks attributable to putative factors associated with HIV-1 transmission, but there is little evidence that individuals from those sub-Saharan countries worst affected by HIV-1 either have more sexual partners or more frequent sexual intercourse than those in countries least affected. 
Under-reporting of AIDS occurred in many countries early on in the pandemic and still continues to some extent. However, more recently, WHO-initiated National AIDS Control programmes have facilitated HIV-1 serosurveillance in most countries. A close relationship between STD and HIV-1 has been demonstrated in numerous studies and the incidence and prevalence of gonorrhoea are often taken to reflect high risk sexual behaviour. However, this may only be partly true in some heterosexual groups in whom factors other than the numbers of unprotected sex acts may be more relevant to the risk of HIV-1 transmission. Thus in Lagos, Nigeria, the prevalence of gonorrhoea has been reported as the highest in the world, ${ }^{11}$ but the numbers of AIDS cases in that country are still very low (184, as of 15.3.92), ${ }^{8}$ suggesting that factors other than sexual behaviour are at least as important in the spread of HIV-1.

One STD syndrome, genital ulcer disease (GUD) has been consistently implicated as a risk factor for acquiring HIV-1. In some populations the proportion of HIV-1 infections attributable to GUD in men may be 75-98\%. ${ }^{12}$ Another independent risk factor for HIV-1 in men is lack of circumcision ${ }^{13}$ which may itself be a risk factor for chancroid, ${ }^{14} 15$ a common cause of GUD in Africa. Analysis of male circumcision practices in Africa indicates a high degree of correlation between the intact foreskin and geographical locations with a high prevalence of HIV-1 infection. ${ }^{1617}$ Furthermore, although data are limited, it would seem that GUD ${ }^{18-20}$ and lack of circumcision ${ }^{1617}$ in men are more common in East, Central and Southern Africa when compared to West Africa and are, with a few exceptions, almost invariably associated with a higher prevalence of HIV-1. These exceptions may be readily explained: thus, in the Ivory Coast male circumcision is not practised universally and HIV-1 infection in men is associated with GUD and lack of circumci$\operatorname{sion}^{21}$; in Ghana three-quarters of AIDS cases are said to be acquired outside the country, most being in female prostitutes returning from neighbouring countries ${ }^{22}$; the high prevalence of GUD in the Gambia, ${ }^{23}$ where male circumcision is almost universal, may reflect partial rather than complete removal of the foreskin. ${ }^{24}$ What is the mechanism that puts uncircumcised men with or without GUD at increased risk of HIV and what interventions could be implemented in this group?

In a study of 73 men in Nairobi, Kenya who acquired a genital ulcer from a single sexual exposure with a prostitute of lower socioeconomic status, $8 \%$ also acquired HIV-1. ${ }^{13}$ Furthermore, a cumulative $43 \%$ of uncircumcised men who acquired an ulcer seroconverted to HIV-1. Clearly these men possessed an abnormality that predisposed to both HIV-1 infection and chancroid, the commonest cause of GUD in Nairobi. The pathogenesis of the GUD (chancroid, syphilis and donovanosis) is still unclear but probably requires an initial minor abrasion of normal skin. In many developing countries such abrasions are common in uncircumcised men with poor standards of genital hygiene and areas of mucosal discontinuity on the penis are present almost constantly. The moist environment beneath the foreskin hinders rapid healing of subpreputial abrasions and also provides an excellent receptacle for the retention of sexually transmitted pathogens. Previous infection with chancroid may be complicated by phimosis, tissue scarring with contraction and deep pitting crevices along the coronal sulcus. The ensuing damage to the superficial subpreputial mucosa, which may be exacerbated if traumatised by sexual intercourse, forms a highly plausible entry point for HIV-1. Conversely, in developed countries where the prevalence of GUD is low and standards of personal hygiene are much higher, such abnormalities are uncommon in uncircumcised men.

Few follow-up studies of men with chancroid have been undertaken but observations from the 1940-50s and even earlier may be relevant to the control of GUD in Africa now. In one study of $1402 \mathrm{UN}$ military personnel with chancroid in Korea in 1952,14, a high incidence of sequelae was recorded. Ulcers at the preputial margin were often destructive leaving a triangular defect in the prepuce and in others, healing resulted in a cicatrix which constricted and narrowed the preputial opening. It was noted that both in this report and in a study of chancroid amongst army personnel in the USA, ${ }^{25}$ that the numbers of cases in blacks was disproportionately greater than whites despite similar exposure risks. Although these studies were conducted amongst army personnel and generally undertaken in a war environment they bear direct comparison with parts of Africa today. The threat of personal injury through everyday violence, as if in a war situation, is a reality in many African countries and may explain a relative relaxation of sexual mores and disregard of STD symptoms in some communities.

Despite interventions directed at all aspects of transmission, the spread of HIV-1 infection continues unabated in many countries. Although the burden of infectious diseases is still a major cause of morbidity and mortality in Africa, control programmes have been successful in reducing the incidence of many diseases. However, little interest has been shown in STDs until relatively recently and many aspects of STD control now merit immediate attention. Ideally an intervention directed towards reducing the spread of HIV-1 and STD in resource-poor settings should be simple, sustainable, acceptable, cheap and effective. Because of the urgent nature of arresting the spread of HIV-1, complex, expensive and in some cases untested interventions are now being considered seriously. Social marketing of drugs, widespread testing for STDs, mass antibiotic treatment and male circumcision are all possible interventions but require further justification and are of questionable 
sustainability. Drug treatments effective for $\beta$-lactamase producing strains of Neisseria gonorrhoeae and Haemophilus ducreyii are expensive and unlikely to be included in national essential drug lists. Few laboratory tests are available for STDs and rapid methods to diagnose the various causes of GUD in the early stages are urgently required. Mass circumcision in men might reduce the incidence of GUD and HIV-1 but the practicality and acceptability of such an intervention has not been evaluated and must be considered doubtful. In the rush to ensure that something be done and seen to be done, is it possible that simple and sustainable interventions have been overlooked?

Specific protective practices for reducing the incidence of STDs in men have included restriction of sexual activities, condoms, systemic and local prophylaxis ${ }^{26}$. During the First and Second World Wars troops exposed to prostitutes ${ }^{27}{ }^{28}$ with high rates of STD were encouraged to attend post coital centres where local prophylaxis was dispensed. Antiseptic mercurial creams ${ }^{27}$, urethral irrigation with protargol and local application of soap and water ${ }^{29}$, an excellent prophylactic against chancroid ${ }^{30}$ and effective even in the presence of skin abrasions, were successful in reducing the incidence of STD in the armed forces. Furthermore, omission of soap and water was identified as the cause of failed prophylaxis for chancroid amongst the American Expeditionary Forces in Paris during the First World War ${ }^{29}$. Although postcoital centres are probably only viable in a war situation, soap and water prophylaxis to improve genital hygiene in uncircumcised men could be promoted in Africa as a component of AIDS prevention programmes. This would involve not only post-exposure washing but, also, and perhaps more importantly, pre-exposure prophylaxis with regular application of soap and water beneath the foreskin.

The practicalities of providing soap and water will vary from country to country and may be impossible in times of drought or in remote rural areas. The ever increasing numbers migrating to cities where large sectors of the population already live in shacks or squatter settlements with poor utility services and a variable water supply are an on-going problem that is undoubtedly accelerating the course of the HIV-1 epidemic. However, increased access to water for personal hygiene could be a feasible objective in these areas if requested as a priority by public health authorities. Where limited State resources preclude provision of water, washing facilities could be provided in the workplace. Employers could be encouraged to improve facilities and their co-operation motivated if made to realise that their work-force could be drastically reduced by the epidemic. Elsewhere this intervention could be introduced initially at sites where core groups of uncircumcised men with GUD seek health care-thus, STD clinics, general practitioners, hospital outpatients and pharmacies could all be targeted. Education would entail practical demonstration of how to retract and clean beneath the foreskin and lengthy explanation would not be required. The concept of HIV-1 transmission through penile abrasions or ulcers is a simple one and would be expected to provide sufficient incentive to improve personal standards of genital hygiene. Furthermore, this message could readily both be passed on from father to son and included as a component of AIDS education in schools. Although the prevalence of GUD in women and therefore the risk of acquiring HIV-1 attributable to GUD may be considerably less than in men ${ }^{12}$, women could play an important role in this intervention by stressing the importance of genital hygiene in their male partners.

The problem of GUD control in countries with significant HIV-1 problems now merits urgent reappraisal in the light of the strong association between GUD, the intact foreskin and acquisition of HIV-1. In many ways the problem has been compounded by the natural history of GUD whereby genital herpes and primary syphilitic chancres resolve spontaneously thereby justifying therapies dispensed by traditional healers and strengthening the belief that medical treatment is not always necessary. In some communities STD are still a source of shame and embarrassment, a perception often reinforced by the attitudes of unsympathetic staff. Late presentation is common and many will have resorted to treatments with little proven therapeutic value from various diverse sources elsewhere. An undoubted benefit of improved genital hygiene would be increased acceptance of patients on a social level by medical and paramedical staff who would, in turn, be better able to communicate and deliver health education more effectively and enthusiastically.

The problem of AIDS in Africa is inextricably linked to poverty and lack of education and at present there are no more obvious available medical solutions than political or economic ones. An intervention such as improving genital hygiene should ideally impinge on the whole population but could not be implemented by medical or healthoriented agencies alone. All agencies involved with assisting developing countries should place AIDS high on their agenda and reassess how their individual expertise could be best deployed against the spread of HIV-1. Agencies involved in improving both access to, and supplies of water, could have a lot more to offer than realised previously.

Although neither the precise timing nor mechanism of female to male transmission of HIV-1 is known, it would seem plausible that infected secretions could be retained beneath the foreskin thereby enhancing viral transmission. Could soap and water prophylaxis have an impact in preventing HIV transmission outside Africa? In Asia and the Indian SubContinent male circumcision is uncommon ${ }^{31}$ and HIV-1 infection is increasing rapidly particularly amongst commercial sex workers 
and their clients. Post-exposure prophylaxis with soap and water could offer some degree of risk reduction in men having unprotected sex with high risk partners.

It must be said that many cases of HIV-1 amongst heterosexuals are transmitted when poor genital hygiene, GUD and lack of circumcision are not relevant. However, the evidence now is that the overwhelming majority of HIV-1 infections in African men are attributable to these factors. Although primary prophylaxis with application of soap and water to the genitalia in uncircumcised men will not be a universal solution for preventing HIV-1 infection, and should not be regarded as a substitute for correct use of condoms, this method may be effective against both chancroid ${ }^{29}$ and syphilis. ${ }^{32}$ The corollary is that by protecting against GUD and penile abrasions, the risk of acquiring HIV-1 by men in high risk core groups will be reduced. This intervention could be evaluated by studying men with GUD and assessing recurrences following a health education programme stressing the importance of both pre- and post-exposure prophylaxis with soap and water. Resources directed at approaching the HIV/STD/GUD problem using those principles of STD control shown to be effective in times of war may offer a more cost effective approach than some of the complex technologies already in operation that are unlikely to be sustainable in the developing world.

1 Prural A, Chacko S, Koch-Weser D. Sexual behaviour, AIDS and poverty in sub-Saharan Africa. Int $\mathcal{F} S T D$ AIDS 1991;2:1-9.

2 O'Farrell N, Windsor I. Sexual behaviour in HIV-1 seropositive men and women in Durban, South Africa. 7 AIDS 1991;4:1258-9.

3 Rowley JT, Anderson RM, Ng T. Reducing the spread of HIV infection in sub-Saharan Africa: some demographic and economic implications. AIDS 1990;4:47-56.

4 Moses S, Plummer FA, Ngugi EN, Nagelkerke NJ, Anzala AO, Ndinya-Achola JO. Controlling HIV in Africa: effectiveness and cost of an intervention in a high-frequency STD transmitter core group. AIDS 1991;5: 407-11.

5 Cameron DW, Ngugi EN, Ronald AR, et al. Condom use prevents genital ulcers in women working as prostitutes. Sex Transm Dis 1991;18:188-91.

6 Laukamm-Josten U, Ocheng D, Mwizarubi BK, Mwaijonga CL, Swai R, Trupin M. HIV and syphilis seroprevalence and risk factors in truckstops and nearby communities in Tanzania. VIII International communities in Tanzania. VIII International
Conference on AIDS, Amsterdam 19-24 July 1992. Abstract POC 4162 .
7 O'Farrell N, Hoosen AA, Coetzee K, van den Ende J. Sexual behaviour in Zulu men and women with genital ulcer disease. Genitourin Med 1992;68:245-8.

8 World Health Organisation Global Statistics. Statistics from the World Health Organisation and the Centres for Disease Control. AIDS 1992;6:1229-33.

9 Department of National Health and Population Development. Second national survey of women attending antenatal clinics, South Africa, October/November 1991. Epidemiological Comments 1992;19:80-92.

10 O'Farrell N, Windsor I. Prevalence of HIV-1 antibodies in recurrent attenders at a sexually transmitted diseases clinic. $S$ Afr Med $¥$ 1988;74:104-5.

11 World Health Organisation. First report of the expert committee on gonococcal infections. Technical Report Series No. 262. Geneva WHO, 1963.

12 Hayes $R$, Schulz KF. What proportion of HIV infections are attributable to genital ulcers in sub-Saharan Africa. are attributable to genital ulcers in sub-Saharan Africa. 19-24 July 1992. Abstract No. MOC 0029.

13 Cameron DW, Simonsen JN, D'Costa LJ, et al. Female to male transmission of human immunodeficiency virus type 1: risk factors for seroconversion in men. Lancet 1989;ii:403-7.

14 Asin J. Chancroid: a report of 1402 cases. American Fournal of Syphilis 1952;36:483-7.

15 Hart G. Factors influencing venereal infection in a war environment. Br $\mathcal{F}$ Venereal Dis 1974; 50:68-72.

16 Moses S, Bradley JE, Nagelkerke NJ, Ronald AR, NdinyaAchola JO, Plummer FA. Geographical patterns of male seroprevalence. Int $\mathcal{F}$ Epidemiol 1990;19:693-7.

17 Bongaarts J, Reining $P$, Way $P$, Conant F. The relationsongaarts J, Reining $P$, Way $P$, Conant $F$. The relation-
ship between male circumcision and $H I V$ infection in African populations. AIDS 1989;3:373-7.

18 Sogbetun AO, Alausa KO, Osoba AO. Sexually transmitted diseases in Ibadan, Nigeria. Br $\mathcal{f}$ Venereal Dis 1977;53:155-60.

19 Willcox RR. Venereal syphilis and other venereal infections in non-venereal treponematoses areas in Africa. Cent Afr ₹ Med 1958;4:432-43.

20 O'Farrell N, Hoosen AA, Coetzee K, van den Ende J. Genital ulcer disease in men in Durban, South Africa. Genitourin Med 1991;67:327-30.

21 Diallo MO, Ackah AN, LaFontaine M-F, et al. HIV-1 and HIV-2 infections in men attending sexually transmitted disease clinics in Abidjan, Cote d'Ivoire. AIDS 1992; 6:581-5

22 Amofah GK. AIDS in Ghana: profile, strategies and challenges. AIDS Analysis Africa 1992;2:5.

23 Mabey DC, Wall RA, Bello CS. Aetiology of genital ulceration in Gambia. Genitourin Med 1987;63:312-5.

24 Pepin J, Quigley $\mathbf{M}$, Todd J, et al. Association between HIV-2 infection and genital ulcer diseases among male sexually transmitted disease patients in the Gambia. AIDS 1992;6:489-93.

25 Greenwald $\mathrm{E}$. Chancroid infection. Treatment and diagnosis. $\mathscr{f} A M A$ 1943;121:9-11.

26 Curran JW. Prevention of sexually transmitted diseases. In: Holmes KK, Mardh P-A, Sparling PF, Wiesner PJ (eds.). Sexually Transmitted Diseases, New York, McGraw Hill 1984;973-91.

27 Campbell DJ. Venereal diseases in the armed forces. $\mathrm{Br} F$ Venereal Dis 1946;22:158-64.

28 Moore JE. The value of prophylaxis against venereal diseases. $\mathcal{F A M A}$ 1920;75:911-5.

29 Moore JE. The diagnosis of chancroid and the effect of prophylaxis upon its incidence in the American Expeditionary Forces. $\mathcal{F}$ Urol 1920;4:169-76.

30 Levin EA. The diagnosis of chancroid. Urologic and Cutaneous Review 1941;45:587-90.

31 Royce R. Does male circumcision prevent HIV infection? In: Mann J, Tarantola DJ, Netter T, eds. AIDS in the In: Mann J, Tarantola DJ, Netter T, eds. AIDS

32 Reasoner MA. The effect of soap on Treponema pallidum. $\mathscr{F A M A}$ 1917;68:973-4. 